\title{
Independent Domination Number in Adaptive Mesh Refinement (AMR)-WENO Scheme Networks
}

\author{
N.Senthurpriya , S.Meenakshi
}

\begin{abstract}
Let $G$ be the graph, consider the vertex set as $V$ and edge set as $E$. If $S$ is the subset of the vertex set $V$ such that $S$ contains vertices which has atleast one neighbor in $V$ that is not in $S$, then $S$ is said to be dominating set of $G$. If the vertex in $S$ is not adjacent to one another, then $S$ is called as the independent dominating set of $G$ and so $i(G)$ represents the independent domination number, the minimum cardinality of an independent dominating set in $G$. In this paper, we obtain independent domination number for triangular, quadrilateral, pentagonal, hexagonal, heptagonal and octagonal networks by Adaptive Mesh Refinement (AMR)-WENO Scheme.
\end{abstract}

Keywords: Adaptive Mesh Refinement (AMR), Domination set, Independent domination number, Independent domination set, WENO Scheme

\section{INTRODUCTION}

In ancient times the ideas of dominating sets, is started with the game of chess in India over 400 years ago, in which we study sets of chess pieces which cover different squares of the chessboard.

Later in the books like Ahrens in 1901 [16], the eight Queens and Five Queens problems again gave the interest in dominating concepts. Finally with the books published by Berge in 1958 [2] and ore in 1962 [9] with the topic in domination gave proper mathematical definition but by 1972 Cockayne and Hedetniemi [3,4] done a same work under domination and began to study it, ultimately published a survey of the results in 1975 and the independent domination number were introduced and it is denoted in the form $i(G)$. This attracted the researchers to work on it. Goddard et al. $[13,15]$, Kostichka [1] and Lam et al. [10] researched much about the independent dominating number in regular graph and cubic graph. Favaron [8] studied the sharp upper bounds for $\boldsymbol{i}(\boldsymbol{G})$ as functions of $n$ and $\delta$ for general graphs and the work was extended by Haviland [7]. Cockayne et al. [5] obtained the product of the $\mathrm{i}(\mathrm{G})$ of a graph and its complement with its upper bound, while Shiu et al. [14] obtained $\mathrm{i}(\mathrm{G})$ of triangle-free graphs and characterizing the extremal graphs with its upper bounds.

\section{DEFINITIONS}

\section{Definition.1 [6] Adaptive Mesh Refinement:}

The AMR technique implemented is a fully conformal method that can work on hybrid meshes, which follows a hierarchical tree based structure. The refinement or

Revised Manuscript Received on December 5, 2019.

Correspondence Author

N.Senthurpriya, Department of mathematics, Vels institute of science technology and advanced studies, Pallavaram, Chennai, India. Email: pspriyasaha@gmail.com

S.Meenakshi, Department of mathematics, Vels institute of science technology and advanced studies, Pallavaram, Chennai, India. Email:meenakshikarthikeyan@yahoo.co.in coarsening is node based and he decision is always taken at the parent level i.e., the data of the initial parent cell is preserved and is always carried over to the number of adaptations done during the process which limits the coarsening to be done only up to the initial mesh level.

\section{Definition.2 [6] WENO Scheme:}

Weighted Essentially Non-Oscillatory Scheme

WENO scheme is actually an approximation procedure which was first introduced by Liu, Osher and Chan in 1994 and later many methods of WENO schemes were introduced. In this paper, we use the above-mentioned networks of different levels which was formed using AMR-WENO Scheme method.

Definition 3. [11] For $v \in V(G)$, the open neighborhood of $v$, denoted as $N_{G}(v)$, is the vertices of the set adjacent with a vertex $v$; and the closed neighborhood of a vertex $v$, denoted by $N_{G}[v]$, is given by $N_{G}(v) \bigcup\{v\}$. For a set $S \subseteq V(G)$, the open neighborhood of a set $S$ is defined by $N_{G}(S)=\bigcup_{v \in S} N_{G}(v)$ and the closed neighborhood of $\mathrm{S}$ is defined as $N_{G}[S]=N_{G}(S) \cup S$. For brevity, we denote $N_{G}(S)$ by $N(S)$ and $N_{G}[S]$ by $N[S]$.

Definition 4. [11] Let $\mathrm{G}$ be a graph with Vertex set $\mathrm{V}$ and edge set $\mathrm{E}$, let $\mathrm{S}$ be the subset of $\mathrm{V}$ such that every vertex in $\mathrm{V}$ which is not in $\mathrm{S}$ must contain at least one neighbor in $\mathrm{S}$. The domination number of $\mathrm{G}$ is denoted by $\gamma(G)$, the minimum cardinality of dominating set of $\mathrm{G}$.

Definition 5. [12] If $S$ is both an independent and dominating set of a graph $G$ then $S$ is said to be an independent dominating set of graph $\mathrm{G}$. The independent domination number is denoted by $i(G)$, the minimum cardinality of an independent dominating set in $\mathrm{G}$.

\section{Theorem 1:}

Let $\mathrm{G}$ be a triangular graph with 3 vertices and 3 edges. Then the independent domination number for AMR by WENO scheme of $\mathrm{G}$ at $n^{\text {th }}$ level is

$$
\mathrm{i}(\mathrm{G})=\left\lceil\frac{\mathrm{n}}{\mathrm{a}}\right\rceil
$$

Proof:

To prove $\mathrm{i}(\mathrm{G})=\left\lceil\frac{\mathrm{n}}{\mathrm{n}}\right\rceil$

Label the vertices of $\mathrm{G}$ as follows:

Label the vertices of $\mathrm{G}$ at $n^{\text {th }}$ level as $\left(u_{1}, v_{1}, w_{1}\right),\left(u_{2}, v_{2}, w_{2}\right), \ldots\left(u_{i}, v_{i}, w_{i}\right)$

And the total number of vertices in each level is given by $n$ times the base network (here the base network is the triangle which has three vertices)

Therefore, this theorem asserts that each level of $G$ has $3 n$ vertices. 
Hence in each level we get the independent domination number as $\left\lceil\frac{\mathrm{n}}{\mathrm{a}}\right\rceil$

Therefore $\mathrm{i}(\mathrm{G})=\left[\frac{\mathrm{n}}{\mathrm{n}}\right\rceil$ at $n^{\text {th }}$ level of $\mathrm{G}$.

For example,
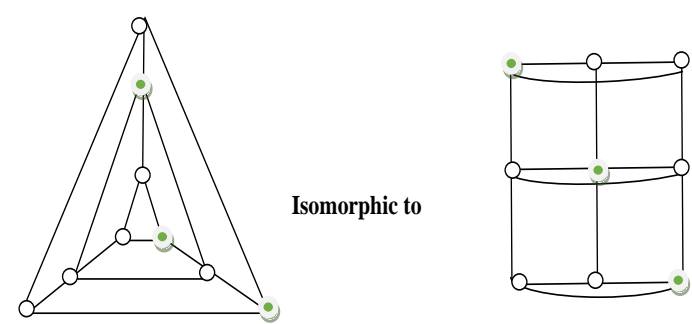

The highlighted vertices represent the independent domination set.

\section{Theorem 2:}

Let $\mathrm{G}$ be a quadrilateral graph with 4 vertices and 4 edges. Then the independent domination number for AMR by WENO scheme of $\mathrm{G}$ at $n^{\text {th }}$ level is $\mathrm{i}(\mathrm{G})=\left\lceil\frac{\mathrm{n}}{4}\right\rceil$

\section{Proof:}

To prove $\mathrm{i}(\mathrm{G})=\left[\frac{\mathrm{n}}{4}\right]$

Label the vertices of $\mathrm{G}$ as follows:

Label the vertices of $\mathrm{G}$ at $n^{\text {th }}$ level as $\left(u_{1}, v_{1}, w_{1}, x_{1}\right),\left(u_{2}, v_{2}, w_{2}, x_{2}\right), \ldots\left(u_{i j}, v_{i}, w_{i j}, x_{i}\right)$

And the total number of vertices in each level is given by $n$ times the base network (here the base network is the quadrilateral which has four vertices)

Therefore, this theorem asserts that each level of $\mathrm{G}$ has $4 \mathrm{n}$ vertices.

Hence in each level we get the independent domination number as $\left\lceil\frac{\mathrm{n}}{4}\right\rceil$

Therefore $\mathrm{i}(\mathrm{G})=\left[\frac{\mathrm{n}}{4}\right\rceil$ at $n^{\text {th }}$ level of $\mathrm{G}$.

For example,
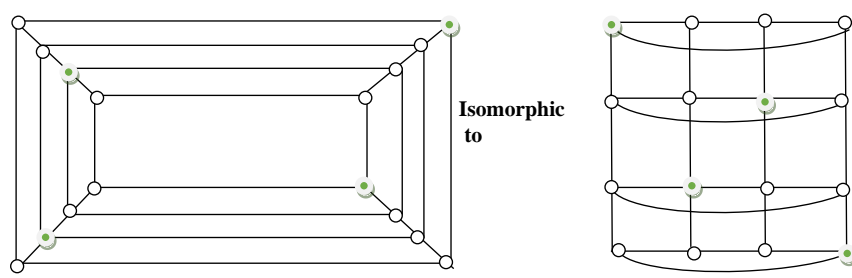

The highlighted vertices represent the independent domination set.

\section{Theorem 3:}

Let $\mathrm{G}$ be a pentagonal graph with 5 vertices and 5 edges. Then the independent domination number for AMR by WENO scheme of $\mathrm{G}$ at $n^{\text {th }}$ level is $\mathrm{i}(\mathrm{G})=\left[\frac{\mathrm{n}}{5}+2\right]$

\section{Proof:}

To prove $\mathrm{i}(\mathrm{G})=\left[\frac{\mathrm{n}}{5}+2\right]$

Label the vertices of $\mathrm{G}$ as follows:

Label the vertices of $\mathrm{G}$ at $n^{\text {th }}$ level as $\left(u_{1}, v_{1}, w_{1}, x_{1} x y_{1}\right),\left(u_{2}, v_{2}, w_{2}, x_{2} v y_{2}\right), \ldots\left(u_{i j}, v_{i}, w_{i}, x_{i}, y_{i}\right)$
And the total number of vertices in each level is given by $n$ times the base network (here the base network is the pentagon which has five vertices)

Therefore, this theorem asserts that each level of $\mathrm{G}$ has $5 \mathrm{n}$ vertices.

Hence in each level we get the independent domination number as $\left[\frac{n}{5}+2\right]$

Therefore $\mathrm{i}(\mathrm{G})=\left[\frac{n}{5}+2\right]$ at $n^{\text {th }}$ level of $\mathrm{G}$.

For example,

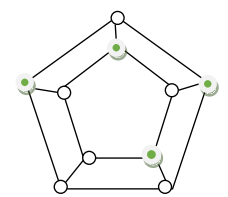

The highlighted vertices represent the independent domination set.

\section{Theorem 4:}

Let $\mathrm{G}$ be a hexagonal graph with 6 vertices and 6 edges. Then the independent domination number for AMR by WENO scheme of $\mathrm{G}$ at $n^{\text {th }}$ level is $\mathrm{i}(\mathrm{G})=\left\lceil\frac{n}{6}+2\right\rceil$

Proof:

To prove $\mathrm{i}(\mathrm{G})=\left[\frac{\mathrm{n}}{6}+2\right\rceil$

Label the vertices of $\mathrm{G}$ as follows:

Label the vertices of $\mathrm{G}$ at $n^{\text {th }}$ level as $\left(u_{1}, v_{1}, w_{1}, x_{1}, y_{1}, \mathbb{Z}_{1}\right),\left(u_{2}, v_{2}, w_{2}, x_{2}, Y_{2}, \mathbb{Z}_{2}\right)$, . . . $\left(u_{i}, v_{i}, w_{i}, x_{i}, y_{i}, z_{i}\right)$

And the total number of vertices in each level is given by $n$ times the base network (here the base network is the hexagon which has six vertices)

Therefore, this theorem asserts that each level of $G$ has $6 n$ vertices.

Hence in each level we get the independent domination number as $\left\lceil\frac{n}{6}+2\right]$

Therefore $\mathrm{i}(\mathrm{G})=\left[\frac{\mathrm{n}}{6}+2\right]$ at $n^{\text {th }}$ level of $\mathrm{G}$.

For example,

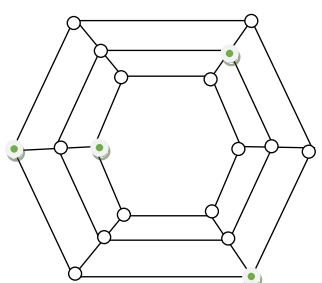

The highlighted vertices represent the independent domination set.

Theorem 5:

Let $\mathrm{G}$ be a heptagonal graph with 7 vertices and 7 edges. Then the independent domination number for AMR by WENO scheme of $\mathrm{G}$ at $n^{\text {th }}$ level is $\mathrm{i}(\mathrm{G})=\left\lceil\frac{2 \mathrm{n}}{\mathrm{g}}\right\rceil$

Proof:

To prove $\mathrm{i}(\mathrm{G})=\left\lceil\frac{2 n}{7}\right\rceil$

Label the vertices of $\mathrm{G}$ as follows: 
Label the vertices of $\mathrm{G}$ at $n^{\text {th }}$ level as $\left(u_{1}, v_{1}, w_{1}, x_{1}, y_{1}, z_{1}, a_{1}\right),\left(u_{2}, v_{2}, w_{2}, x_{2}, y_{2}, z_{2}, a_{2}\right)$, . . $\left(u_{i}, v_{i j}, w_{i}, x_{i}, y_{i}, z_{i}, a_{i}\right)$

And the total number of vertices in each level is given by $n$ times the base network (here the base network is the heptagon which has seven vertices)

Therefore, this theorem asserts that each level of $\mathrm{G}$ has $7 \mathrm{n}$ vertices.

Hence in each level we get the independent domination number as $\left\lceil\frac{2 n}{7}\right\rceil$

Therefore $\mathrm{i}(\mathrm{G})=\left\lceil\frac{2 n}{7}\right\rceil$ at $n^{\text {th }}$ level of $\mathrm{G}$.

For example,

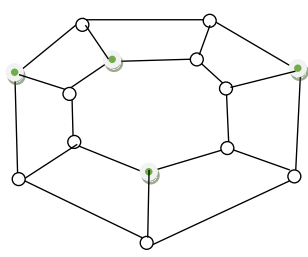

The highlighted vertices represent the independent domination set.

\section{Theorem 6:}

Let $\mathrm{G}$ be a octogonal graph with 8 vertices and 8 edges. Then the independent domination number for AMR by WENO scheme of $\mathrm{G}$ at $n^{\text {th }}$ level is

$\mathrm{i}(\mathrm{G})=\left\lceil\frac{\mathrm{n}}{4}\right\rceil$

\section{Proof:}

To prove $\mathrm{i}(\mathrm{G})=\left[\frac{\mathrm{n}}{4}\right\rceil$

Label the vertices of $\mathrm{G}$ as follows:

Label the vertices of $\mathrm{G}$ at $n^{\text {th }}$ level as $\left(u_{1}, v_{1}, w_{1}, x_{1}, y_{1}, z_{1}, a_{1}, b_{1}\right),\left(u_{2}, v_{2}, w_{2}, x_{2}, J_{2}, z_{2}, a_{2}, b_{2}\right)$. . . $\left(u_{i}, v_{i j}, w_{i}, x_{i}, Y_{i}, z_{i j}, a_{i}, b_{i}\right)$

And the total number of vertices in each level is given by $n$ times the base network (here the base network is the octagon which has eight vertices)

Therefore, this theorem asserts that each level of $\mathrm{G}$ has $8 \mathrm{n}$ vertices.

Hence in each level we get the independent domination number as $\left\lceil\frac{n}{4}\right\rceil$

Therefore $\mathrm{i}(\mathrm{G})=\left[\frac{\mathrm{n}}{4}\right\rceil$ at $n^{\text {th }}$ level of $\mathrm{G}$.

For example,

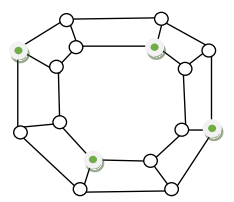

\section{CONCLUSION}

In this paper, we find the $\mathrm{i}(\mathrm{G})$ for triangular, quadrilateral, pentagonal, hexagonal, heptagonal and octagonal networks by Adaptive Mesh Refinement (AMR)-WENO Scheme. And hence in future papers we extend $\mathrm{i}(\mathrm{G})$ for other based networks by AMR-WENO Scheme

\section{REFERENCES}

1. A. V. Kostochka, The independent domination number of a cubic 3 -connected graph can be much larger than its domination number, Graphs Combin., 9 (1993) pp. 235-237.

2. C. Berge . Theory of Graphs and its Applications, Methuen, London. (1962)

3. E. J. Cockayne, and S. T. Hedetniemi, Independent graphs, Congr. Numer., X (1974) 471-491.

4. E. J. Cockayne and S. T. Hedetniemi. Towards a theory of domination in graphs, Networks 7 (1977) 247-261

5. E. J. Cockayne, O. Favaron, H. Li, and G. MacGillivray, The product of the independent domination numbers of a graph and its complement, Discrete Mathematics, 90 (1991) 313-317.

6. Harshavardhana Srinivasan and Panagiotis Tsoutanis, Adaptive mesh refinement techniques for high-order finite- volume WENO scheme, conference paper, January 2016

7. J. Haviland. Upper bounds for independent domination in regular graphs, Discrete Math., 307 (2007) 2643-2646.

8. O. Favaron. Two relations between the parameters of independence and irredundance, Discrete Math., 70 (1988) 17-20.

9. O. Ore, Theory of graphs, Amer. Math. Soc. Transl., 38 (1962) pp. 206-212.

10. P. Lam, W. Shiu and L. Sun On independent domination number of regular graphs, Discrete Mathematics Combin, 202 (1999) 135-144.

11. S. Ao, E. G. Cockayne MacGillivray and C. M. Mynhardt, Domination critical graphs with higher independent domination numbers, J. Graph Theory, 22 (1996) 9-14

12. T.W. Haynes, S.M. Hedetniemi, S.T. Hedetniemi and M.A. Henning, Power domination in graphs applied to electrical power networks, SIAM Journal on Discrete Mathematics, 15(4) (2002) 519-529.

13. Wayne Goddard and Michael A.Henning, Independent domination in graphs: a survey and recent results, Discrete Mathematics, 313(2013)839-854

14. W. C. Shiu,X. Chen and W. H. Chan, Triangle-free graphs with large independent domination number, Discrete Optim., 7 (2010) pp. 86-92.

15. W.Goddard, M. Henning,J. Lyle and J. Southey. On the independen domination number of regular graphs, Ann. Comb., 16 (2012) 719-732.

16. Wilhelm Ahrens (1st ed.), Mathematische Unterhaltungen und Spiele, Teubner, Leipzig (1901)

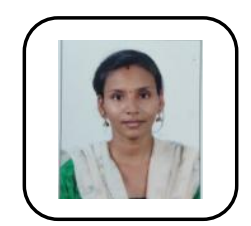

\section{AUTHORS PROFILE}

N.Senthurpriya Research scholar at Vels institute of science technology and advanced studies, pallavaram, Chennai-117, published a paper in The international journal of analytic and experimental modal analysis ISSN NO:0886-9367 under the topic Independent domination number in cycle necklace graph Vol XI Issue X October/2019.

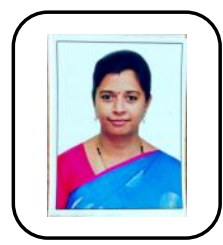

S.Meenakshi Associate Professor Department of Mathematics Vels institute of science technology and advanced studies, pallavaram, Chennai-117. 